\title{
Argon Plasma: A New Approach for the Effective Treatment of Inflammatory Acne Vulgaris and Enlarged Pores in Asian Patients
}

\author{
Sung Bin $\mathrm{Cho}^{1,2}$ \\ Hyun-Jo Kim ${ }^{1,3}$ \\ Heesu Kim \\ Kwang $\mathrm{Ho} \mathrm{Yoo}^{1}$
}

\footnotetext{
${ }^{1}$ Department of Dermatology and Cutaneous Biology Research Center, International St. Mary's Hospital, Catholic Kwandong University College of Medicine, Incheon, Korea

${ }^{2}$ Kangskin Dermatology Clinic, Seoul, Korea

${ }^{3}$ CNP Skin Clinic, Cheonan, Korea
}

\begin{abstract}
Acne vulgaris is a common chronic inflammatory disease of the pilosebaceous units that usually begins in adolescence. This condition can lead to serious psychosocial problems, and can have adverse effects on an individual's quality of life. Enlarged pores are a common complication of acne. Although a variety of approaches can be used to treat inflammatory acne vulgaris and enlarged pores, the disadvantages of a long healing time, dyspigmentation, prolonged erythema, and pain make these treatments unsatisfactory, particularly in Asians. Gas molecules, such as nitrogen, helium, and argon, can be ionized into a high-energy state of matter called "plasma." While plasma has recently been shown to elicit several therapeutic effects in dermatologic areas, there is no consensus regarding the optimal implementation for the treatment of inflammatory acne vulgaris and enlarged pores. In this study, two patients were treated with acne vulgaris and enlarged pore using argon plasma. After a few sessions, the patients showed almost complete improvement with no remarkable side effects or recurrence over the duration of a few months. The results suggest that argon plasma can be used effectively and safely for the treatment of acne vulgaris, particularly inflammatory lesions and enlarged pores, in Asian patients.
\end{abstract}

\section{Key words}

Plasma; Argon; Acne vulgaris; Enlarged pore
Received December 14, 2018

Accepted December 17, 2018

\section{Correspondence}

Kwang Ho Yoo

Department of Dermatology, International St. Mary's Hospital, Catholic Kwandong University College of Medicine, 25 Simgok-ro, Seo-gu, Incheon 22711, Korea

Tel.: +82-32-290-3141

Fax: +82-32-290-3142

E-mail: psyfan9077anaver.com

(c) Korean Society for Laser Medicine and Surgery

(c) This is an open access article distributed under the terms of the Creative Commons Attribution NonCommercial License (http://creativecommons.org/ licenses/by-nc/4.0) which permits unrestricted noncommercial use, distribution, and reproduction in any medium, provided the original work is properly cited. 


\section{INTRODUCTION}

Acne vulgaris is a common, chronic, multifactorial inflammatory dermatosis that clinically presents with open or closed comedones and inflammatory papules, pustules, nodules, or cysts.1 Major contributing factors for developing acne vulgaris include proliferation of Propionibacterium acnes (P. acnes), dermal inflammation, abnormal follicular keratinocyte proliferation and desquamation, and increased sebum production. ${ }^{1}$ In addition to various topical and systemic agents for treating acne vulgaris, energy-delivering systems have been used as additive or alternative therapeutic modalities. ${ }^{1-6}$ Among these, light and laser sources have been deemed to modify or destroy the key pathogenic factors in the development of inflammatory acne lesions. ${ }^{1-6}$ Moreover, early intervention using energy-delivering systems has been described as reducing the risks of post-acne erythema, dyspigmentation, and scarring. ${ }^{4,5}$

Plasma is the fourth state of matter, consisting of charged electrons and ions. ${ }^{7.8}$ For various medical purposes, plasma can be generated from inert gaseous sources, including nitrogen, helium, argon, and am ambient air, by stripping electrons from atoms using ultra-high frequency generators. ${ }^{9,10}$ Argon plasma has been used medically to elicit antibacterial, antiviral, antifungal, and antipruritic effects. ${ }^{11-13}$ Moreover, research has shown that low temperature argon plasma induces wound repair process by activating nuclear transcription factor- $\mathrm{kB}$ pathway to upregulate cyclinD1 expression and promote fibroblast proliferation. ${ }^{14}$

In this report, we describe two Korean female patients with acne vulgaris and enlarged pores who were effectively treated with pulsed delivery of argon plasma.

\section{CASE REPORT}

\section{Case 1}

A 27-year-old Korean female visited our clinic presenting with erythematous to brownish papular lesions with enlarged pores on the forehead, nose, perinasal area, and lateral cheeks. The patient had been treated with oral and topical medications, chemical peeling, pulsed dye laser, quasi-long neodymium ( $\mathrm{Nd}$ ):yttrium aluminum garnet (YAG), invasive bipolar radiofrequency using microneedle electrodes, and carbon dioxide and erbium:YAG fractionated lasers at our clinic. However, the clinical outcomes thereof were unsatisfactory.

After obtaining written informed consent, the patient was treated with a single session of argon plasma treat- ment using a plasma generator Pladuo $^{\mathrm{TM}}$; Shenb Co., Ltd., Seoul, Koreal that generates plasma from argon and nitrogen gas sources and delivers pulses thereof to the skin. Argon plasma was prepared by loading $0.48-\mathrm{ml}$ argon gas/pulse, and a microwave generator with a frequency of $2.45 \mathrm{GHz}$ was used to activate inert argon gas. The other treatment settings included a pulse energy of $0.8 \mathrm{~J}$, a pulse duration of $12 \mathrm{msec}$, a nozzle diameter of 5 $\mathrm{mm}$, and a distance from the nozzle's tip to the skin of 10 $\mathrm{mm}$. After cleansing the patient's face with a mild soap and $70 \%$ ethanol, open and closed comedones were gently extracted without pretreatment with topical anesthetic cream. Thereafter, the entire face was treated with a single pass of argon plasma treatment, and acne lesions or enlarged pores were treated with an additional two to three more argon plasma pulses for a total shot count of approximately 900 shots. The treated areas were cooled with icepacks immediately after treatment. No prophylactic systemic or topical corticosteroids, antibiotics, or antiviral agents were prescribed. The patient was recommended to avoid excessive sun exposure.

Two months after the treatment, the patient exhibited marked reductions in inflammatory acne lesions and post-acne erythema and hyperpigmentation (Fig. 1). Furthermore, enlarged pores, skin texture, and overall skin tone were improved, and the patient was very satisfied with the results. Pain during the treatment was tolerable without the use of topical anesthetic cream, and most of the post-treatment erythema spontaneously disappeared within two hours. Furthermore, no remarkable major side effects, including itching, crusts, oozing, burn, worsening of inflammatory acne lesions or folliculitis, prolonged edema or erythema, postinflammatory hyperpigmentation, and scarring, were encountered.

\section{Case 2}

A 30-year-old Korean female visited our clinic presenting with erythematous to brownish papular lesions with enlarged pores on the forehead, nose, perinasal area, and medial cheeks. The patient had been treated with oral and topical medications, chemical peeling, and various energy-delivering devices at other clinics; however, the clinical outcomes thereof were unsatisfactory. After obtaining written informed consent, the patient was treated with three sessions of argon plasma treatment using a plasma generator (Pladuo ${ }^{\mathrm{TM}}$ ) at 2-week intervals. Argon plasma was prepared by loading $0.44-\mathrm{ml}$ argon gas/pulse and was activated with a microwave generator. The other treatment settings included a pulse energy of $0.75 \mathrm{~J}$, a pulse duration of $11 \mathrm{msec}$, a nozzle diameter of $5 \mathrm{~mm}$, 

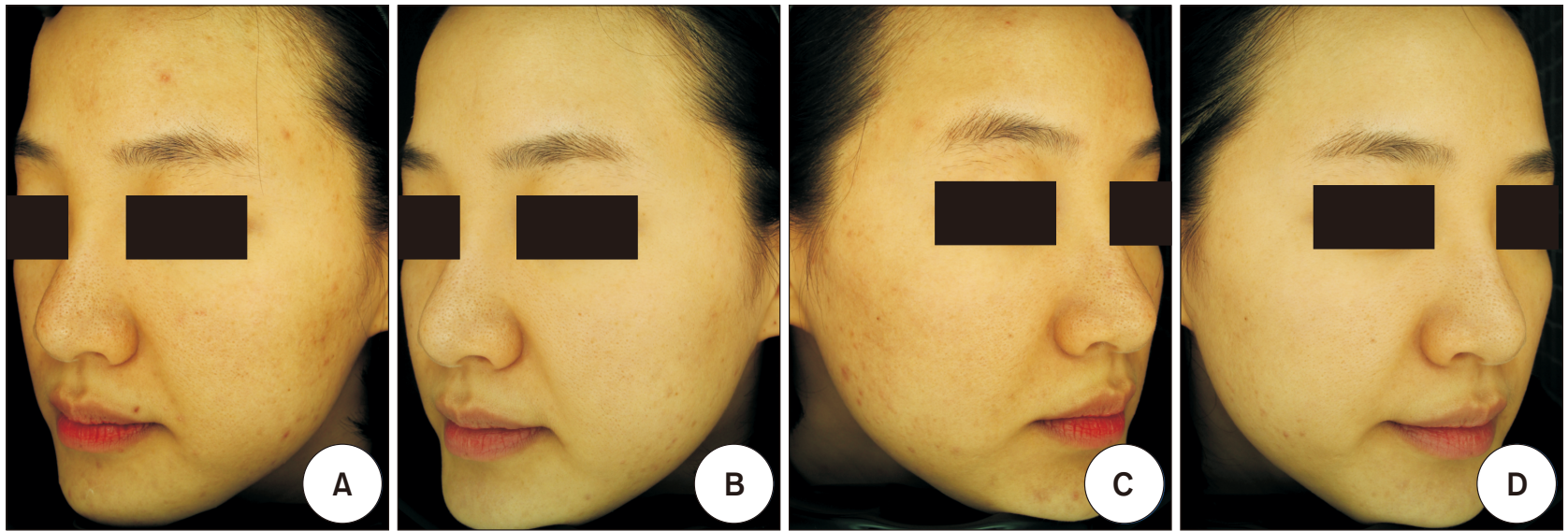

Fig. 1. Case 1. Photographs of a 27-year-old Korean female patient (A, C) at baseline and (B, D) two months after one session of argon plasma treatment with a loading volume of $0.48-\mathrm{ml}$ argon gas/pulse, a pulse energy of $0.8 \mathrm{~J}$, a pulse duration of $12 \mathrm{msec}$, a nozzle diameter of $5 \mathrm{~mm}$, and a distance from the nozzle's tip to the skin of $10 \mathrm{~mm}$. Photographs were taken under normal light exposure. (A, B) Left oblique view, (C, D) right oblique view.
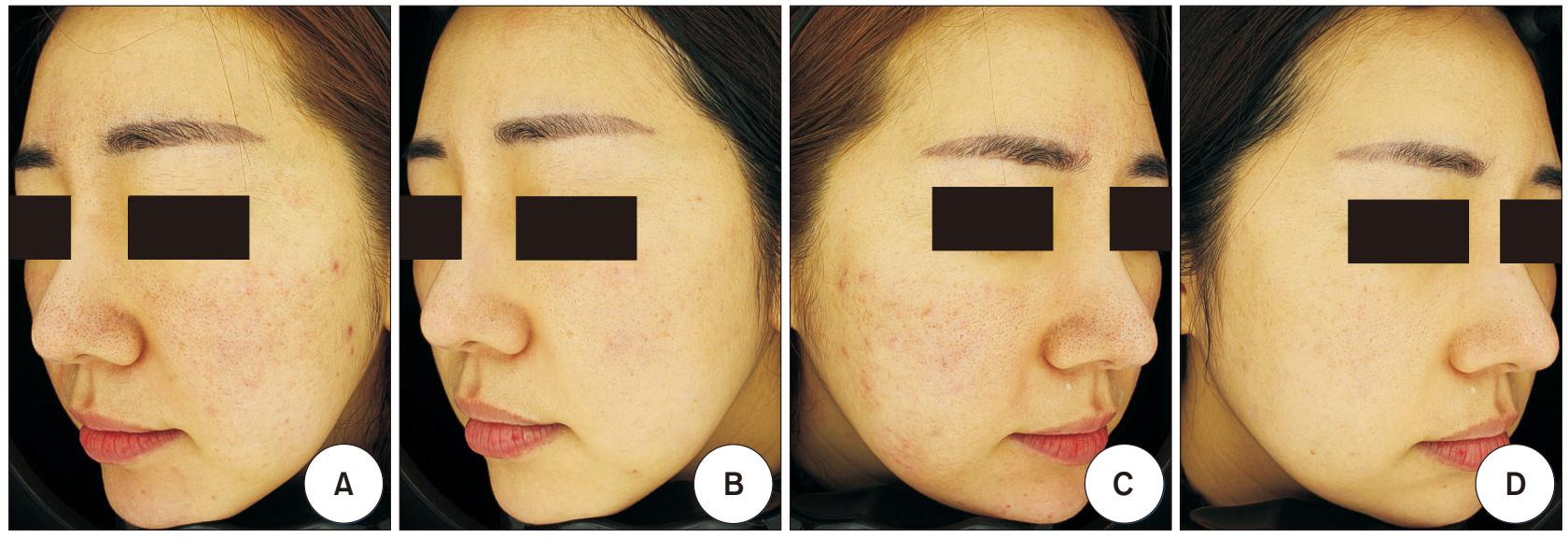

Fig. 2. Case 2. Photographs of a 30-year-old Korean female patient (A, C) at baseline and (B, D) one month after three sessions of argon plasma treatment at 2 -week intervals. Treatment settings included a loading volume of $0.44-\mathrm{ml}$ argon gas/pulse, a pulse energy of $0.75 \mathrm{~J}$, a pulse duration of $11 \mathrm{msec}$, a nozzle diameter of $5 \mathrm{~mm}$, and a distance from the nozzle's tip to the skin of $10 \mathrm{~mm}$. Photographs were taken under normal light exposure. (A, B) Left oblique view, (C, D) right oblique view.

and a distance from the nozzle's tip to the skin of $10 \mathrm{~mm}$. The patient's face was first cleansed with a mild soap and $70 \%$ ethanol, after which the entire face was treated with two or three passes of argon plasma treatment with a total shot count of approximately $700-900$ shots/session without pretreatment with topical anesthetic cream. The treated areas were cooled with icepacks immediately after treatment. No prophylactic systemic or topical corticosteroids, antibiotics, or antiviral agents were prescribed. The patient was recommended to avoid excessive sun exposure.

One month after the treatment, she exhibited marked reductions in inflammatory acne lesions and post-acne erythema and hyperpigmentation (Fig. 2). Ultraviolet pho- tographs, which were taken at an identical setting using an imaging tool (Mark-Vu ${ }^{\circledR}$; VIEWERMEDI, Seoul, Korea), revealed marked reductions in protoporphyrin IX by $P$. acnes (Fig. 3). Enlarged pores, skin texture, and overall skin tone had improved, and the patient was very satisfied with the results. Pain during the treatment was tolerable without the use of topical anesthetic cream, and most of the post-treatment erythema spontaneously disappeared within two hours. No remarkable major side effects, including itching, crusts, oozing, burn, worsening of inflammatory acne lesions or folliculitis, prolonged edema or erythema, postinflammatory hyperpigmentation, and scarring, were encountered. 

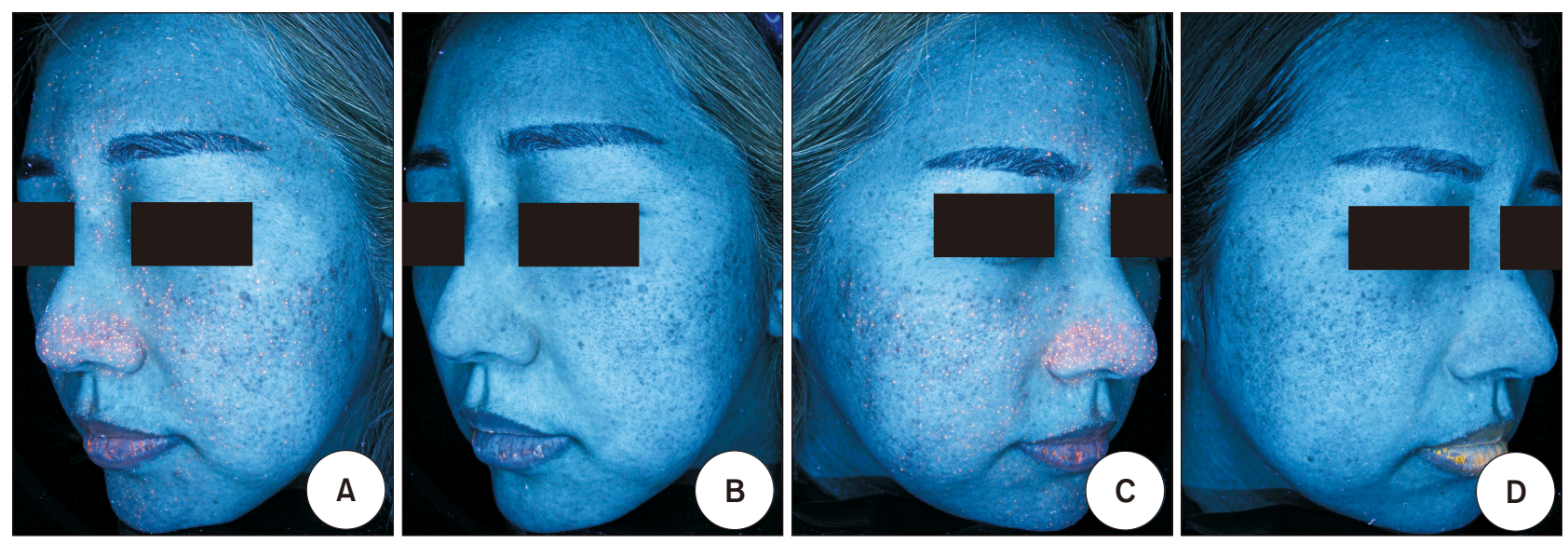

Fig. 3. Case 2. Photographs of a 30-year-old Korean female patient (A, C) at baseline and (B, D) one month after three sessions of argon plasma treatment at 2-week intervals. Pinkish fluorescent areas in the centrofacial skin at baseline represent protoporphyrin IX by Propionibacterium acnes. Photographs were taken under ultraviolet light exposure. (A, B) Left oblique view, (C, D) right oblique view.

\section{DISCUSSION}

Among various treatment modalities, those including lasers and light-based and energy-based technology have become an integral component of the therapeutic arsenal for treating acne vulgaris and enlarged pores, particularly for patients who fail to respond to or do not tolerate other treatments, including oral and topical medications. While improvements can be achieved with several medical devices, they carry different risks of adverse side effects, including delayed erythema, persistent hyperpigmentation, prolonged healing times, and pain, limiting the application of medical devices, especially in Asians. ${ }^{4,15}$

In our study, the treatment of argon plasma showed several advantages, based on our personal experience, over conventional other modalities in treating both inflammatory acne vulgaris and enlarged pores. First, and most important, was in minimizing the development of antibiotic resistance, which is associated with disruption of the normal flora, bacterial resistance among other organisms, and increased rates of upper respiratory infection and pharyngitis. ${ }^{16}$ Oral antibiotics are the most common systemic agent prescribed for the treatment of acne. However, antibiotic resistance is a growing problem across medicine and rates of antibiotic resistance among isolates of $P$. acnes have been rising, including to tetracycline-class antibiotics. Accordingly, researchers have called for limiting the use of oral antibiotics in the treatment of acne. ${ }^{17}$ Meanwhile, studies have indicated that plasma holds profound bactericidal and fungicidal properties in vitro, and a prospective randomized controlled trial has shown argon plasma to be efficacious and safe in decreasing bacterial load in chronic wounds. ${ }^{11,12,18}$
As seen in Fig. 3, argon plasma markedly reduced protoporphyrin IX from P. acnes as an antibacterial effect. Therefore, we believe that the use of argon plasma can be effective in treating inflammatory acne lesions by destroying $P$. acnes while avoiding the potential for antibiotic resistance.

The second advantage of using argon plasma is that, in addition to inflammatory acne lesions, enlarged pores can also be improved. The appearance of enlarged pores has been linked to the severity and the duration of inflammation occurring in acne lesions. Therefore, an early and adequate treatment regimen to reduce the inflammation of acne lesions may be advisable to prevent enlarged pores. ${ }^{1,15}$ Interestingly, plasma treatment promotes the healing process and collagen remodeling, which are associated the increased expression of transforming growth factor- $\beta$, in the skin. ${ }^{8,18}$ Recently, the relevant mechanism of collagen synthesis has been suggested: 120to 180-second plasma treatment may be related to upregulated expression of wound healing-related factors, including vascular endothelial growth factor, fibroblast growth factor, heparin-binding epidermal growth factorlike growth factor, and interleukin-6, which are responsible for angiogenesis and proliferation. ${ }^{19}$ Histology analysis further revealed faster re-epithelialization, collagen deposition, less inflammation, and a complete skin structure of the epidermis and dermis layer in the plasma-treated groups, compared with the control groups. ${ }^{19}$ Another study described decreases in matrix metalloproteinase-3 levels and increases in laminin levels upon immunohistochemical study after micro-plasma treatment..$^{20}$ Therefore, in our study, we utilized argon plasma to promote re-epithelialization and new collagen synthesis to improve 
the appearance of enlarged pores and skin texture.

In conclusion, because well-designed studies to evaluate the efficacy of procedures utilizing argon plasma versus traditional medical therapies are lacking and standardized regimens have not been agreed upon, they should not be considered first-line treatments for inflammatory acne vulgaris and enlarged pores. Nevertheless, our results provide evidence that argon plasma is not only an effective and safe for the treatment of inflammatory acne vulgaris but also can improve enlarged pores, even in Asian patients. Further controlled investigations are needed to compare and optimize the efficacy and safety of argon plasma treatment for acne vulgaris and enlarged pores.

\section{ACKNOWLEDGEMENTS}

This research was supported by a Biomedical Science Scholarship Grant from the Department of Medicine, Catholic Kwandong University College of Medicine (2018).

We would like to thank Bora Kim (Shenb Co., Ltd., Seoul, Koreal and Min Choi (Shenb Co.) for their assistance with technical support. We would also like to thank Anthony Thomas Milliken, ELS, at Editing Synthase (https://editingsynthase.com) for his help with the editing of this manuscript.

\section{REFERENCES}

1. Zaenglein AL, Pathy AL, Schlosser BJ, Alikhan A, Baldwin HE, Berson DS, et al. Guidelines of care for the management of acne vulgaris. J Am Acad Dermatol 2016;74:945-73.e33.

2. Bitter P Jr. Acne treatment with 3-step broadband light protocol. J Drugs Dermatol 2016;15:1382-8.

3. Seaton ED, Charakida A, Mouser PE, Grace I, Clement RM, Chu AC. Pulsed-dye laser treatment for inflammatory acne vulgaris: randomised controlled trial. Lancet 2003;362:1347-52.

4. Wiznia LE, Stevenson ML, Nagler AR. Laser treatments of active acne. Lasers Med Sci 2017;32:1647-58.

5. Tong $L X$, Brauer JA. Lasers, light, and the treatment of acne: a comprehensive review of the literature. J Drugs Dermatol 2017;16:1095-102.

6. Ito Y, Cho SB. Modified 3-step broadband light treatment for inflammatory acne vulgaris and post-acne erythema in Asian patients. Med Lasers 2017;6:102-6.

7. Bogle MA, Arndt KA, Dover JS. Evaluation of plasma skin regeneration technology in low-energy full-facial rejuvenation.
Arch Dermatol 2007;143:168-74.

8. Heinlin J, Zimmermann JL, Zeman F, Bunk W, Isbary G, Landthaler $M$, et al. Randomized placebo-controlled human pilot study of cold atmospheric argon plasma on skin graft donor sites. Wound Repair Regen 2013;21:800-7.

9. Kilmer S, Semchyshyn N, Shah G, Fitzpatrick R. A pilot study on the use of a plasma skin regeneration device (Portrait PSR3) in full facial rejuvenation procedures. Lasers Med Sci 2007;22:101-9.

10. Elsaie ML, Kammer JN. Evaluation of plasma skin regeneration technology for cutaneous remodeling. J Cosmet Dermatol 2008;7:309-11.

11. Heinlin J, Isbary G, Stolz W, Zeman F, Landthaler M, Morfill G, et al. A randomized two-sided placebo-controlled study on the efficacy and safety of atmospheric non-thermal argon plasma for pruritus. J Eur Acad Dermatol Venereol 2013;27:324-31.

12. Wiegand C, Beier O, Horn K, Pfuch A, Tölke T, Hipler UC, et al. Antimicrobial impact of cold atmospheric pressure plasma on medical critical yeasts and bacteria cultures. Skin Pharmacol Physiol 2014;27:25-35.

13. Weyandt GH, Benoit S, Becker JC, Bröcker EB, Hamm H. [Controlled layered removal of anogenital warts by argon-plasma coagulation]. J Dtsch Dermatol Ges 2005;3:271-5. in German.

14. Liu JR, Xu GM, Shi XM, Zhang GJ. Low temperature plasma promoting fibroblast proliferation by activating the NF-kB pathway and increasing cyclinD1 expression. Sci Rep 2017;7:11698.

15. Alexiades M. Laser and light-based treatments of acne and acne scarring. Clin Dermatol 2017;35:183-9.

16. Barbieri JS, Spaccarelli N, Margolis DJ, James WD. Approaches to limit systemic antibiotic use in acne: systemic alternatives, emerging topical therapies, dietary modification, and laser and light-based treatments. J Am Acad Dermatol. In press 2018.

17. Choi SH, Seo JW, Kim KH. Comparative study of the bactericidal effects of indocyanine green- and methyl aminolevulinatebased photodynamic therapy on Propionibacterium acnes as a new treatment for acne. J Dermatol 2018;45:824-9.

18. Lan T, Xiao Y, Tang L, Hamblin MR, Yin R. Treatment of atrophic acne scarring with fractional micro-plasma radio-frequency in Chinese patients: a prospective study. Lasers Surg Med 2018;50:844-50.

19. Cheng KY, Lin ZH, Cheng YP, Chiu HY, Yeh NL, Wu TK, et al. Wound healing in streptozotocin-induced diabetic rats using atmospheric-pressure argon plasma jet. Sci Rep 2018;8:12214.

20. Shao PL, Liao JD, Wong TW, Wang YC, Leu S, Yip HK. Enhancement of wound healing by non-thermal N2/Ar micro-plasma exposure in mice with fractional-CO2-laser-induced wounds. PLoS One 2016;11:e0156699. 